\title{
Application of Digital Image Correlation to Cement Paste ${ }^{\dagger}$
}

\author{
Evin Dildar Dzaye ${ }^{1,2, *}$, Geert De Schutter ${ }^{2}$ and Dimitrios Aggelis 1 \\ 1 MeMC, Vrije Universiteit Brussel, 1050 Brussels, Belgium; dimitrios.aggelis@vub.be \\ 2 Magnel Laboratory for Concrete Research, Ghent University, B-9052 Ghent, Belgium; \\ geert.deschutter@ugent.be \\ * Correspondence: Evin.dildar.a.dzaye@vub.be; Tel.: +32-(0)2-629-29-36 \\ + Presented at the 18th International Conference on Experimental Mechanics (ICEM18), Brussels, Belgium, \\ 1-5 July 2018.
}

Published: 12 June 2018

\begin{abstract}
In this paper digital image correlation (DIC) has been applied to study the deformation process of cementitious material at very early age. After mixing of cement-based materials, the cement hydration process begins. Consequently, the ongoing chemical reactions result in a 3D deformation process (shrinkage). The mechanism affecting the very early age hydration as well as specifically the deformation behavior of cementitious materials is a challenging topic. In view of that, it is essential to determine the significant effect of concrete hardening process on the deformation progression at different stages. The technique of DIC is highly sensitive and allows for the first time in literature an accurate and non-contact optical monitoring of the shrinkage of fresh cementitious material. The displacement of the surface is measured by correlating the different digital images taken at different ages after mixing of the material. The system enables a 3D observation that allows a deeper understanding of the deformation progression. The surface displacement determined by DIC-software (Vic-Snap 2010) is compared to the displacement measured by Linear variable differential transformer (LVDT) sensors for calibration purposes. DIC system realizes a more precise method avoiding the effect of self-weight of the traditional sensor. The purpose of this work is to check the sensitivity as well as the effectiveness of DIC technique, to characterize and better understand the 3D deformation process of fresh cementitious materials.
\end{abstract}

Keywords : digital image correlation; nondestructive technique; cement-based materials

\section{Introduction}

Concrete exhibits volume changes that has detrimental effects on the long-term properties. The microstructure of concrete stiffens with the time that causes in a concrete volume changes. As a result, concrete afflict volume reduction caused by the loss of moisture which is called shrinkage. There are several phenomena that contribute to concrete shrinkage such as chemical hardening due to hydration process [1], drying process due to changes of pore water content and thermal variations. For this reason, the essential evaluation of concrete deformation when it shrinks becomes complex. Recently several studies have applied Digital Image Correlation (DIC) to monitor concrete displacement, as DIC can monitor full-field and has the sensitivity to record non-uniform displacement distributions. Maurouc et al. [2] examined cracking of a coating mortar caused by restraint of drying shrinkage. 2D-DIC was applied to capture the mapping of shrinkage strain and to study the evolution of micro-cracking pattern on mortar surface during several days. DIC measurement was compared with classically LVDT sensors and a good concordance between both methods was obtained. In another study, surface cracking due to water transfer process and stress distribution during concrete drying is evaluated using DIC [3]. Lagier et al. [4] applied 2D-DIC to 
determine cracking due to drying process. They quantified the incompatibility effects between cement paste and aggregates related to drying shrinkage. Yang et al. [5] determined the effect of aggregate size and volume on drying shrinkage of concrete and mortar by 3D-DIC. The non-uniform strain distribution increased with drying time.

This study is focused on the 3D-DIC displacement distribution in cementitious materials in fresh stage (few minutes after casting) to understand in depth material's drying process. Displacement of concrete causes cracks that is one of the most significant defects in concrete and decreases the service life of concrete. The vertical deformation (settlement) in the plastic state may also cause plastic settlement cracks and is associated to "bleed water" pushed to the free surface. Settlement is connected to bleeding and segregation that have a negative effect on post-curing concrete properties, therefore its presence should be assessed. In this study, the settlement built due to shrinkage will be visualized and studied. The DIC mapping of vertical deformation will be compared to classic point measurement methods. This way, similar to the work of Slowik et al. [6], casting and curing processes can be adjusted to eliminate shrinkage formation.

\section{Brief Overview of DIC Principles}

DIC is an optical, non-contact, full-field surface measurement technique. A pair of high resolution cameras builds a stereoscopic vision system. The cameras are synchronized and triggered to simultaneously capture images at a constant time interval. DIC images are analyzed in postprocessing stage to detect differences at the surface between reference (undeformed or healthy state) and deformed surface state. Any variation at the image intensity can be associated to deformation. In this direction, the sample surface is covered with randomly distributed white and black speckles. The DIC post-processing software measures the grey intensity of local zones (subsets) [7]. Displacement in space translated to movement of the speckle pattern is perceived as change on the subset grey intensity degree compared to a reference stage [8].

DIC measurement accuracy depends on the speckle pattern quality controlled by black-white contrast, sharpness and speckles size and distribution. There is no standardized and optimized method to apply the speckles, therefore there is a variety of different application procedures in literature, such as marked pen [7], spray paint, screen printing and airbrush gun [9]. Application of DIC on fresh cementitious media implies a challenging speckle pattern application since the speckles might move due to bleed water or get absorbed on the wet surface.

\section{Material and Experimental Setup}

\subsection{Cementitious Material}

Specimens of cement paste were made using normal Portland cement (Cement CEM I 52.5N and water). The cement paste was prepared with a water to binder ratio of 0.45 . The material was prepared in a laboratory concrete mixer and was mixed for $3 \mathrm{~min}$ at low speed. Afterward, the material was poured into metallic mold of size $150 \times 150 \times 150 \mathrm{~mm}$ (internal dimensions) in a single layer (Figure 1a,b). All inside surfaces of the metallic mold were lubricated with a release agent. 


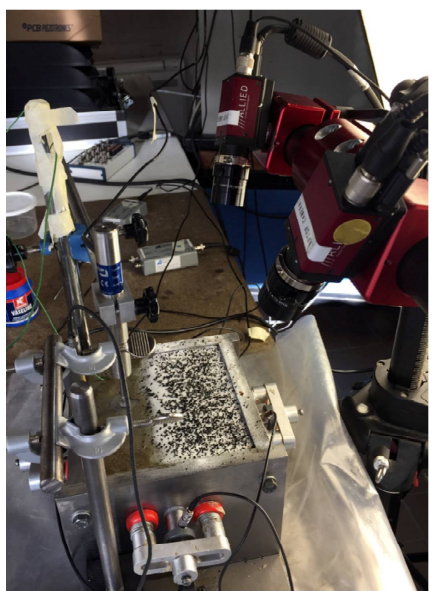

(a)

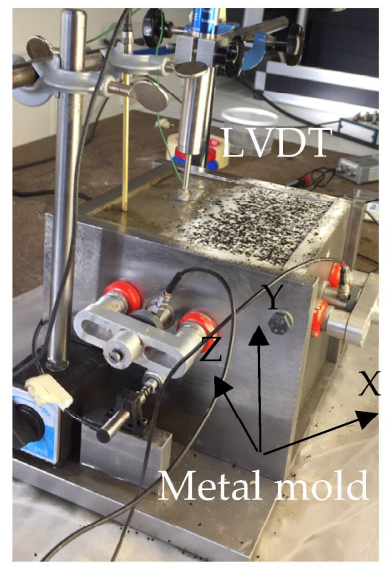

(b)

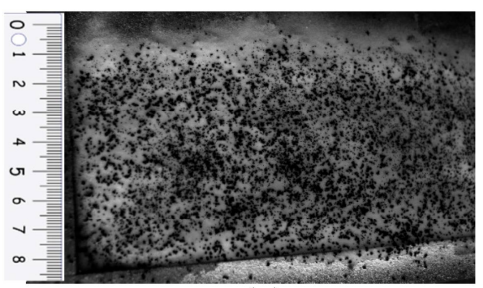

(c)

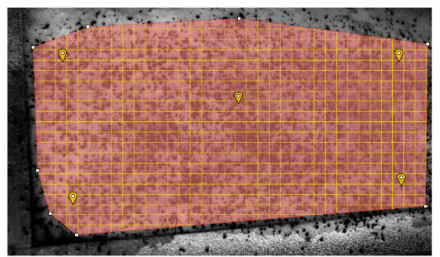

(d)

Figure 1. (a) Experimental setup including DIC system; (b) representation of the setup including LVDT sensor and DIC coordinate; (c) Sample surface covered with speckle pattern; (d) Sample surface with subset size.

\subsection{Experimental Setup}

A Linear Variable Differential Transformer (LVDT) is embedded vertically into the cement paste in order to measure vertical displacement. The LVDT is attached to a metal support and is placed at the middle of the free-surface. LVDT tip touched a $20 \times 20 \mathrm{~mm}$ metallic wire lattice that prevents tip penetration as well as floating on the surface.

A pair of digital cameras (AVT Stingray, allied vision technologies GMBH, Stadtroda, Germany) was placed above the mold as shown in Figure 1a. The cameras have resolution of 2504 by 2056 pixels. During the measurement, smooth white light was placed above the mortar surface to provide light at the whole monitoring area. The lens type was CNG 1.4/12-0902 with a focal length of $12 \mathrm{~mm}$. The cameras were set at a distance of $200 \mathrm{~mm}$ from the specimen surface. All analyses were performed with $61 \times 61$ subsets with a step size of 10 between subset centers (Gaussian subset weights, optimized 8-tap interpolation with normalized squared differences) to obtain full-field displacement measurements. The average speckle size is $1.2 \mathrm{~mm}$. Images are captured every $120 \mathrm{~s}$ by the use of VicSnap 2010. The analysis of the data is performed by Vic-3D 7 provided by Correlated Solution. The field of view is $115 \times 63 \mathrm{~mm}$.

The specimen surface is covered by a powder speckle pattern (white layer obtained by applying Aluminum oxide and black speckles using carbon) immediately after casting the concrete into the mold. First a layer of Aluminum oxide powder and later the carbon particles were applied through a sieve. Only the half of the specimen surface is covered with speckle pattern since in the middle of the free surface the LVDT is placed and beyond that point the LVDT shadow affects the DIC measurement. Consequently, DIC can visualize an area at which the left, right and bottom sides (as shown in Figure 1d) are attached to the metallic mold and the top side stands in the middle area of the sample. The latter should be highlighted since the boundary conditions are not identical for all four side of the analysis area. The analysis is done considering an axis system shown in Figure $1 \mathrm{~b}$. The displacement vectors $\mathrm{U}, \mathrm{V}, \mathrm{W}$ in $\mathrm{mm}$ are calculated at $\mathrm{X}, \mathrm{Y}, \mathrm{Z}$ directions respectively. DIC monitoring started few minutes after casting. Reference starting point $(0 \mathrm{~min})$ is considered the time of casting and the duration of the experiment was approximately $2800 \mathrm{~min}$ ( 2 days). Moreover, one Linear Variable Differential Transformer (LVDT) was applied vertically on the sample surface to measure the settlement at the specimen surface.

\section{Results and Discussion}

In order to detect settlement, DIC vertical displacement ( $\mathrm{W}$ in $\mathrm{mm}$ ) captured at different curing stages are presented in Figure 2. Settlement (in $\mathrm{mm} / \mathrm{m}$ ) is calculated as displacement $\mathrm{W}$ divided by the nominal specimen depth. After casting (Time $0 \mathrm{~min}$ ) and during the early plastic state, water 
concentrates at the top surface (known as 'bleed water') and evaporates in time. As a result, plastic shrinkage triggers displacement and volume loss in the early periods. Increased bleed water in some regions modifies the speckle pattern in plastic state not allowing the analyzes. $30 \mathrm{~min}$ after casting $\mathrm{W}$ displacement at cement free surface increases in a high rate as shown in Figure 3a. Beyond $60 \mathrm{~min}$, a transition from plastic to setting state takes place indicating concrete hardening. At hardening state (representatively in 1000 and 2830 min, Figure 2), settlement effect extends further, but in lower rate this time.

Settlement exhibits its highest value at top left edge of the field of view, standing for the specimen middle zone. It is concluded that displacement is not uniformly distributed, hence settlement cannot be a homogenous spatial phenomenon. It is shown that bleed water movement controls the settlement evolution. The consolidation process of the particle structure and the evolution of the cement paste microstructure decreases the settlement rate at later stages.

It should be highlighted that DIC, compared to traditional contact sensors, measures the settlement in contactless mode, therefore the sensor cannot affect the measurement process, e.g., by increased settlement measured value due to self-weight of the sensor. Based on the observed 3D fullfield DIC maps, it is evident that a point measurement of settlement is not sufficient to describe settlement. The fact that settlement of a point at the surface is influenced by its elevation compared to the surrounding areas, as visually presented in Figure 23D W maps, is investigated further. Figure $3 a$ presents the settlement obtained by DIC the points with the highest (P0), the lowest (P1) (points indicated in Figure 3c) and total average area are compared to classical LVDT point measurement. In all four curves the settlement reaches a stable value $100 \mathrm{~min}$ after monitoring indicating the end of plastic stage and the start of hardening process. Beyond this time, the range is significantly wide between point $\mathrm{P} 0(-10.7 \mathrm{~mm} / \mathrm{m})$ and $\mathrm{P} 1(-2.6 \mathrm{~mm} / \mathrm{m})$. While the point measurement of LVDT presents a settlement of $-8.9 \mathrm{~mm} / \mathrm{m}$ and the average DIC measurement presents a settlement $-6.6 \mathrm{~mm} / \mathrm{m}$ after $450 \mathrm{~min}$, see Figure $3 \mathrm{a}$.

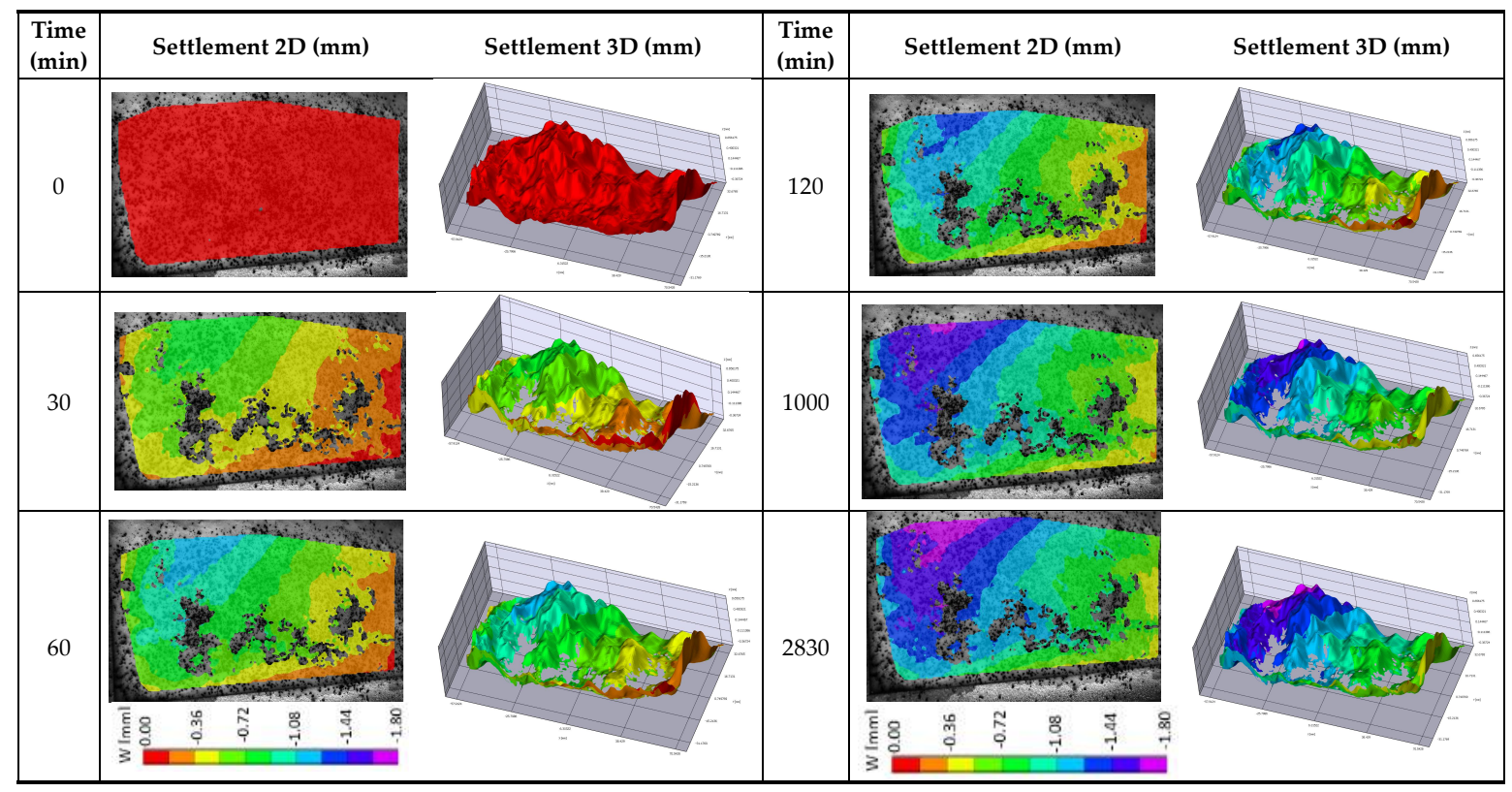

Figure 2. 2D and 3D-DIC surface displacement settlement at discrete monitoring stages of curing. 

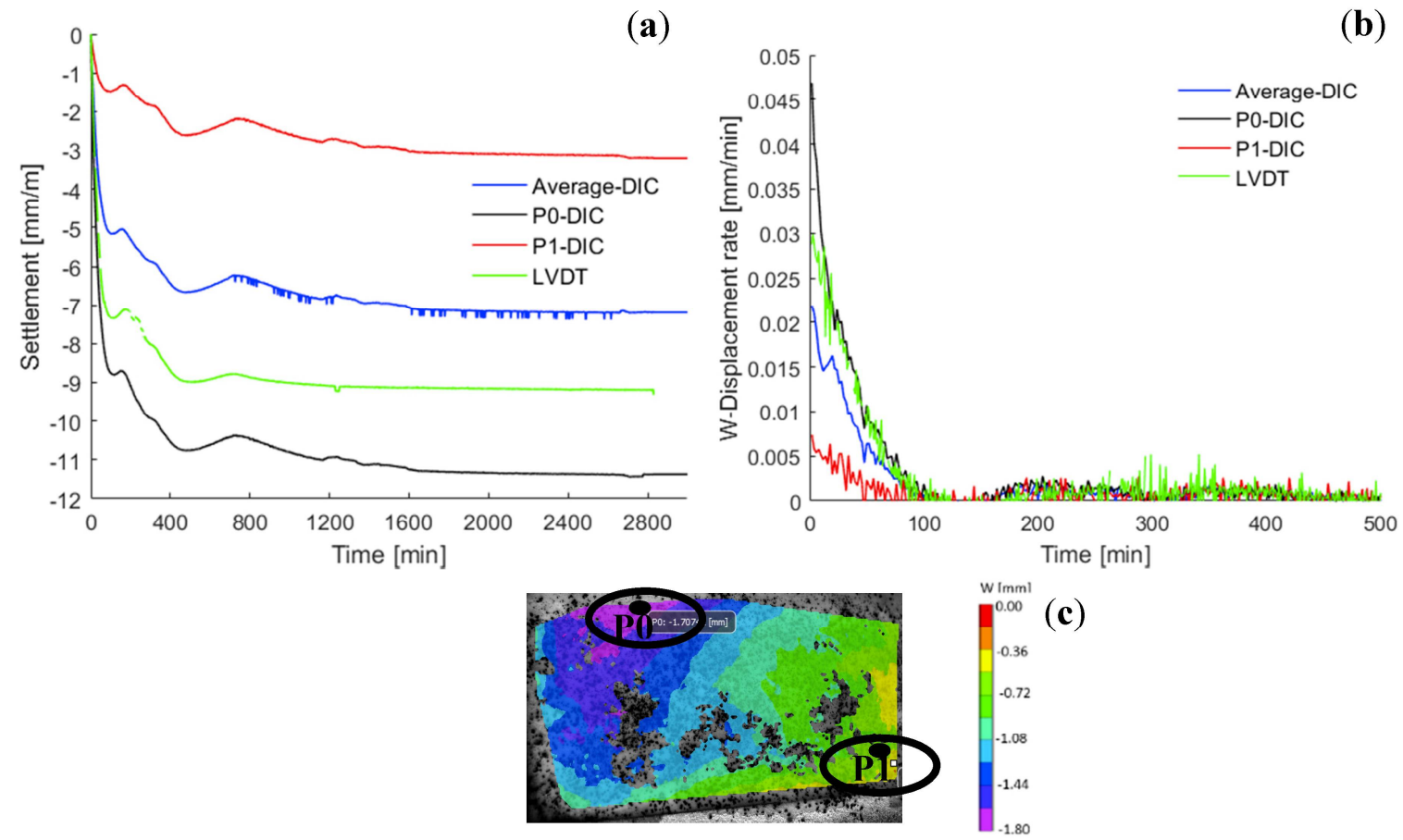

(c)

Figure 3. (a) surface settlement of DIC and LVDT; (b) W settlement rate versus time; (c) points (P0, P1 and total average area) of DIC measurement.

The difference in settlement between LVDT and DIC measurement in the absolute value is attributed to the fact that DIC considers the total surface area and LVDT monitors the settlement at a point of the surface. It is evident that no robust conclusion can be obtained when considering only point analysis. This is an evidence for the significant enhancement offered by the global analysis. Using DIC the variation in settlement in different points is readily available while the average value on the surface can be regarded as a global measurement.

The $\mathrm{W}$ displacement rate in time is shown for point $\mathrm{P} 0, \mathrm{P} 1$, average of the total area and LVDT in Figure $3 \mathrm{~b}$. In this case, the settlement at each location is accompanied by a comparable change of the displacement rate. Point P0 with the highest settlement indicates the highest displacement rate, while point P1 with the lowest settlement indicates the lowest displacement rate.

\section{Conclusions}

In this study, it has been revealed that 3D-DIC realizes measuring settlement evolution of fresh cementitious materials immediately after casting. Settlement is observed on the specimen surface indicating the hardening process by DIC and LVDT point measurement: a non-uniform settlement distribution is obtained by 3D-DIC, while classical LVDT considers only a point measurement. The effective technique of 3D-DIC realizes monitoring the variation in settlement in different points that allows a deeper studying of concrete setting and hardening process.

Acknowledgments: Financial support of the Research Foundation Flanders (FWO-Vlaanderen) through Projects No. G012315N is gratefully acknowledged.

\section{References}

1. Wieslaw, K. Cement and Concrete Chemistry, 1st ed.; Springer: Krakow, Poland, 2014.

2. Mauroux, T.; Benboudjema, F.; Turcry, P.; Aït-Mokhtar, A.; Deves, O. Study of cracking due to drying in coating mortars by digital image correlation. Cem. Concr. Res. 2012, 42, 1014-1023.

3. Maruyama, I.; Sasano, H. Strain and crack distribution in concrete during drying. Mater. Struct. 2014, 47, $517-532$. 
4. Lagier, F.; Jourdain, X.; De Sa, C.; Benboudjema, F.; Colliat, J.B. Numerical strategies for prediction of drying cracks in heterogeneous materials: Comparison upon experimental results. Eng. Struct. 2011, 33, 920-931.

5. Chen, Y.; Wei, J.; Huang, H.; Jin, W.; Yu, Q. Application of 3D-DIC to characterize the effect of aggregate size and volume on non-uniform shrinkage strain distribution in concrete. Cem. Concr. Compos. 2018, 86, 178-189.

6. Slowik, V.; Schmidt, M.; Fritzsch, R. Capillary pressure in fresh cement-based materials and identification of the air entry value. Cem. Concr. Compos. 2008, 30, 557-565.

7. Sutton, M.A.; Orteu, J.-J.; Schreier, H.W. Image Correlation for Shape, Motion and Deformation Measurements, Basic Concepts, Theory and Applications; Springer: New York, NY, USA, 2009.

8. Lecompte, D.; Smits, A.; Bossuyt, S.; Sol, H.; Vantomme, J.; Van Hemelrijck, D.; Habraken, A.M. Quality assessment of speckle patterns for digital image correlation. Opt. Lasers Eng. 2006, 44, 1132-1145.

9. Barranger, Y.; Doumalin, P.; Dupré, J.C.; Germaneau, A. Digital Image Correlation accuracy: Influence of kind of speckle and recording setup. EPJ Web Conf. 2010, 6, 31002.

(C) 2018 by the authors. Licensee MDPI, Basel, Switzerland. This article is an open access article distributed under the terms and conditions of the Creative Commons Attribution (CC BY) license (http://creativecommons.org/licenses/by/4.0/). 